\title{
Proton decay matrix elements in $2+1$ domain-wall fermion
}

Eigo Shintani* (RBC-UKQCD collaboration)

RIKEN-BNL Research Center, Brookhaven National Laboratory, Upton, NY 11973, USA

E-mail: shintani@riken.jp

We compute proton decay matrix element in lattice QCD with $2+1$ flavor dynamical domainwall fermions. This calculation is targeted on the matrix element for $p \rightarrow(\pi, K, \eta)+l$ relevant to the prediction of grand unified theories (GUTs). We evaluate the complete sets of twelve matrix elements related to $p \rightarrow(\pi, K, \eta)$ process involving dimension-six operators violating baryon number conservation. We use a direct extraction from three-point function in a relatively large volume $\left(2.7 \mathrm{fm}^{3}\right)$ and light quark mass $\left(m_{s} / 6\right.$ to $\left.m_{s}\right)$ in the isospin limit. The systematic uncertainties associated with lattice artifacts and finite quark mass correction are controllable by using chiral fermions and the direct extraction method. This result gives an improved bound of proton lifetime for GUT models.

XXIX International Symposium on Lattice Field Theory

July $10-162011$

Squaw Valley, Lake Tahoe, California

\footnotetext{
${ }^{*}$ Speaker.
} 


\section{Introduction}

Proton decay is one of the unique prediction of the Grand Unified Theories (GUTs). GUTs [1] naturally predicts nucleon decay involving heavy particles, e.g. X and Y gauge bosons [1] or colortriplet Higgs multiplet [2,3], exchanges with both color and flavor degree of freedom below GUTs scale $M_{\mathrm{GUTs}} \sim 10^{15-17} \mathrm{GeV}$. Recently SuperKamiokande experiment reports the new lower limit of partial proton lifetime for $p \rightarrow e^{+} \pi^{0}$ channel as $8.2 \times 10^{33}$ year [4]. This is already close to verge of allowed maximum bound ( $\sim 10^{35}$ year) of SUSY-GUTs. Fixing the uncertainties of hadronic effect is one of the important check of whether minimal SUSY-GUTs are already ruled out or not. Since non-perturbative computations of matrix element in lattice QCD are the most reliable methodology to quantify the hadronic contribution, it may provide more accurate information for searching GUTs prediction in the present experiments and feature.

In this proceedings we present calculation of proton decay matrix element using the direct method in full QCD with 2+1 flavor dynamical domain-wall fermion in larger lattice volume $(2.7 \mathrm{fm})^{3}$ and small quark mass (which is $1 / 6$ strange quark mass) and near physical strange quark mass at small lattice spacing ( $a \simeq 0.11 \mathrm{fm}$ ). In our setup the serious systematic uncertainties which might have been included in the previous calculations can be controlled: i) Lattice artifact, thanks to chiral symmetry of the domain-wall fermion on the lattice, $O(a)$ error, where $a$ is lattice spacing, is highly suppressed and there is no need for counter term to remove such lattice artifacts as appeared in Wilson fermion case [5]. ii) Quark mass correction, direct calculation does not need to use the intermediate effective models to evaluate matrix element at the physical point. iii) Full QCD, we take into account the dynamical quark effects in matrix elements, and the dynamical quark contribution to individual matrix elements is estimated from the comparison with previous quenched results [6].

\section{Proton decay matrix element}

\subsection{Effective Lagrangian and matrix element}

We calculate the transition matrix element from nucleon (proton and neutron, $N=p, n$ ) initial state to allowed (pseudoscalar, $P S=(\pi, K, \eta)$ ) meson and lepton final state from dimension six operators

$$
\left\langle P S(\vec{p}), l(\vec{q})\left|\left[\bar{l}^{c} \mathscr{O}^{\Gamma \Gamma^{\prime}}\right]\right| N(\vec{k})\right\rangle=-\sum_{s= \pm 1 / 2} \bar{v}_{l}^{c}(q, s)\left\langle P S ; \vec{p}\left|\mathscr{O}^{\Gamma \Gamma^{\prime}}\right| N ; \vec{k}, s\right\rangle,
$$

including three-dimensional momenta: $\vec{p}$ for final pseudoscalar, $\vec{k}$ for initial nucleon and $\vec{q}=\vec{p}-\vec{k}$ for final lepton which is determined from momentum conservation. Summation in the above equation is taken with both spin $s= \pm 1 / 2$ and Dirac spinor. Amplitude $v_{l}(q, s) \equiv\langle 0|l| l(\vec{q}, s)\rangle$ denotes the wave function of on-shell lepton state at momentum $\vec{q}$ with spin $s$ and $\alpha$ spinor component. Furthermore the matrix element in the quark sector can be generally divided into relevant form factor $W_{0}\left(q^{2}\right)$ and irrelevant one $W_{q}\left(q^{2}\right)$ as

$$
\left\langle P S ; \vec{p}\left|\mathscr{O}^{\Gamma \Gamma^{\prime}}\right| N ; \vec{k}, s\right\rangle=P_{\Gamma^{\prime}}\left[W_{0}^{\Gamma \Gamma^{\prime}}\left(q^{2}\right)-i \not \phi W_{q}^{\Gamma \Gamma^{\prime}}\left(q^{2}\right)\right] u_{N}(k, s),
$$

up to $O\left(m_{l} / m_{N}\right) . W_{0}, W_{q}$ depend on the renormalization scale and square of four-dimensional momentum transfer $q=k-p$. Using on-shell condition, the total matrix element as shown in 
Eq.(2.1) is given by

$$
\begin{aligned}
\sum_{s, \alpha} \bar{v}_{l}^{c}(q, s)\left\langle P S ; \vec{p}\left|\mathscr{O}^{\Gamma \Gamma^{\prime}}\right| N ; \vec{k}, s\right\rangle & =\sum_{s} \bar{v}_{l}^{c}(q, s) P_{\Gamma^{\prime}}\left[W_{0}^{\Gamma \Gamma^{\prime}}\left(q^{2}\right)-i q W_{q}^{\Gamma \Gamma^{\prime}}\left(q^{2}\right)\right] u_{N}(k, s) \\
& =\left(\bar{v}_{l}(q), u_{N}(k)\right)_{\Gamma^{\prime}} W_{0}^{\Gamma \Gamma^{\prime}}(0)+O\left(m_{l} / m_{N}\right),
\end{aligned}
$$

with $i q v_{l}=m_{l} v_{l}$ and $W_{q} / m_{N} \simeq W_{0}$ [6]. Since $m_{l}^{2}=-q^{2}$ is much smaller than nucleon mass squared in the case of $l=e, v$, we set $q^{2}=0$ and ignore the second term. Eventually $W_{0}(\mu, 0)$ is the only relevant form factor to the proton decay matrix element.

\subsection{Lattice set up}

To obtain the matrix element we make use of the ratio of three-point function of (nucleon$O^{\Gamma L}$-meson) with two-point function of nucleon and meson. Such ratio is defined as

$$
R_{3}\left(t, t_{1}, t_{0} ; p, m_{N}, \mathscr{P}\right)=\frac{\operatorname{tr}\left[\mathscr{P}\left\langle J_{P S}\left(\vec{p}, t_{1}\right) \widetilde{\mathscr{O}}^{\Gamma \Gamma^{\prime}}(-\vec{p}, t) J_{N}\left(\overrightarrow{0}, t_{0}\right)\right\rangle\right]}{\left\langle J_{P S}(\vec{p}, t) J_{P S}(-\vec{p}, t)\right\rangle \operatorname{tr}\left[P_{4}\left\langle J_{N}(\overrightarrow{0}, t) J_{N}\left(\overrightarrow{0}, t_{0}\right)\right\rangle\right]} \sqrt{Z_{P S} Z_{N}},
$$

with interpolating field for pseudoscalar $J_{P S}=\bar{q} \gamma_{5} q$ and nucleon $J_{N}$ as in [6]. Note that the operator of nucleon interpolating field is not uniquely determined, and actually we see that two possible operators formed as $J_{N}=\left(u^{T} C \gamma_{5} d\right) u,\left(u^{T} C \gamma_{4} \gamma_{5} d\right) u$ which have same quantum number as proton state. Numerical comparison of results will be shown in the next section. Three-quark operator in momentum space is defined as

$$
\widetilde{\mathscr{O}}^{\Gamma \Gamma^{\prime}}(-\vec{p}, t)=\sum_{\vec{x}} e^{-i \vec{p} \vec{x}}\left(q^{T} C P_{\Gamma} q\right) P_{\Gamma^{\prime}} q(\vec{x}, t) .
$$

In this calculation we use $\vec{n}=(1,0,0),(1,1,0)$ for meson and $\vec{k}=0$ for nucleon in three-point function. $Z_{P S, N}$ indicates the amplitude of overlap of the interpolating field to on-shell state with Dirac spinor normalized by $\bar{u}_{N}(k, s) u_{N}\left(k, s^{\prime}\right)=2 m_{N} \delta_{s s^{\prime}}$. Taking an enough large time separation of meson-operator $\left(t_{1}-t\right)$ and operator-nucleon $\left(t-t_{0}\right)$ in order to suppress the other excited contamination than an asymptotic state of pseudoscalar and nucleon and using two different projection matrices $\mathscr{P}=P_{4}, i P_{4} \gamma_{j}$ to extract form factor from three point function by solving linear equation of

$$
R_{3}\left(p, m_{N}, P_{4}\right)=W_{0}^{\Gamma \Gamma^{\prime}}\left(q^{2}\right)-i q_{4} W_{q}^{\Gamma \Gamma^{\prime}}\left(q^{2}\right), \quad R_{3}\left(p, m_{N}, i P_{4} \gamma_{j}\right)=q_{j} W_{q}^{\Gamma \Gamma^{\prime}}\left(q^{2}\right),
$$

$W_{0}$ and $W_{q}$ can be simultaneously obtained. Note that $-q^{2}=q_{4}^{2}+\vec{q}^{2}=-\left(m_{N}-E_{P S}\right)^{2}+\vec{p}^{2}$, with $q_{4}=i\left(m_{N}-E_{\pi}\right)$ in Minkovski space-time.

We use gauge configurations with dynamical domain-wall fermions of $2+1$ flavor at Iwasaki gauge action in lattice size $24^{3} \times 64$ at $\beta=2.13$ which corresponds to $a^{-1}=1.73(3)$ [7]. This is the same ensemble as previous indirect method study [8]. Boundary condition is periodic for gauge field, and spatially periodic and temporally anti-periodic for fermion field. We use four different unitary quark masses in chiral extrapolation, and one unitary and one partially quenched strange-quark masses in the study of strange quark mass dependence for final $K^{0, \pm}$ state. Explicit chiral symmetry breaking due to finite fifth dimensional lattice as $L_{s}=16$ is small in this ensemble [7] (residual mass is $m_{\text {res }} \simeq 3 \times 10^{-3}$ ), and indeed we take into account residual mass-shift in the 
chiral extrapolation. When computing two-point and three-point function on the lattice, we always use gauge invariant Gaussian source and sink. Nucleon source-point and pseudoscalar sink-point for three-point function are set to fixed $t_{1}=5$ and $t_{0}=27$ individually, and we make operator position in three-point function constructed by sequential source method move between this range. The variation of spacial momentum is $a \vec{p}=(\pi / 12,0,0),(\pi / 12, \pi / 12,0)$. Note that at the lightest quark mass $\left(m_{u d}^{\text {sea }}=0.005\right)$ to increase statistics we further take average over two different source time slices, 5 and 37, for each gauge ensembles.

\section{Results of proton decay matrix element}

\subsection{Plateau region of $W_{0}$}

Figure 1 show the behavior of $W_{0}^{R L}$ of $p \rightarrow \pi^{0}$ channel obtained by taking the trace with spinprojection matrix at each time slices at four different quark masses. Plateau region seems to be around $13 \leq t \leq 20$ at four quark masses. We thus set a fitting region in $13 \leq t \leq 20$ even in the other pseudoscalar channel. In this figure we also compare $W_{0}^{R L}$ with two different nucleon interpolating operators, $\left(q^{T} C \gamma_{4} \gamma_{5} q\right) q$ and $\left(q^{T} C \gamma_{5} q\right) q$. We see that the signal of $W_{0}^{R / L L}$ obtained from their operators are well consistent, and furthermore the time dependence is strongly correlated among them. To obtain $W_{0}$ with constant function, we perform simultaneous fit of two Jackknife data with same fitting range. Compared to individual results, the statistical error of combined results is slightly improved. We use such combined results in the later analysis. To make extrapolation to physical kinematics where $q^{2}=0$ and physical pion and Keon mass, we try to use two different procedures; one is that by using the global fit with a function that depends on both quark mass and $q^{2}=0, W_{0}$ at physical point is obtained straightforwardly. Second is that $W_{0}$ at physical point is given by using the chiral extrapolation of data after taking the $q^{2}=0$ limit with linear function individually. Here the physical point where is given in [7] corresponds to $m_{u d}^{\text {phys }}=0.001385$ and $m_{s}^{\text {phys }}=0.03785$.

\subsection{Extrapolation to physical point}

In [6] they employed the simple linear function for global fitting over square of momentum $q^{2}$ and quark mass. For comparison we first follow their analysis in a similar way. In our case we use the following fitting function:

$$
\begin{aligned}
F_{W_{0}}\left(\pi^{0}, \eta\right) & =A_{0}+A_{1}\left(m_{u d}+m_{\mathrm{res}}\right)+A_{2} q^{2}, \\
F_{W_{0}}(K) & =B_{0}+B_{1}\left(m_{u d}+m_{\mathrm{res}}\right)+B_{2}\left(m_{s}+m_{\mathrm{res}}\right)+B_{3} q^{2},
\end{aligned}
$$

with fitting parameters $A_{i}$ and $B_{i}$. We use four different quark masses, two different strange quark masses and the lowest two spacial momenta, and therefore total number of data points is eight for $\pi$ and $\eta$ or sixteen for $K$ final state. Since $\chi^{2} /$ dof is less than 1.5 for all possible matrix elements, it turns out that the simple linear function as described in Eq.(3.1) and (3.2) is in good agreement with lattice data using simulation points. In the physical point $\left(m_{u d}+m_{\mathrm{res}} \rightarrow m_{u d}^{\text {phys }}, m_{s}+m_{\mathrm{res}} \rightarrow m_{s}^{\text {phys }}\right.$ and $q^{2} \rightarrow 0$ ).

Second we first perform the extrapolation or interpolation to $q^{2}=0$ with linear function and then take a chiral extrapolation to physical quark mass. In this analysis we can visibly look over 

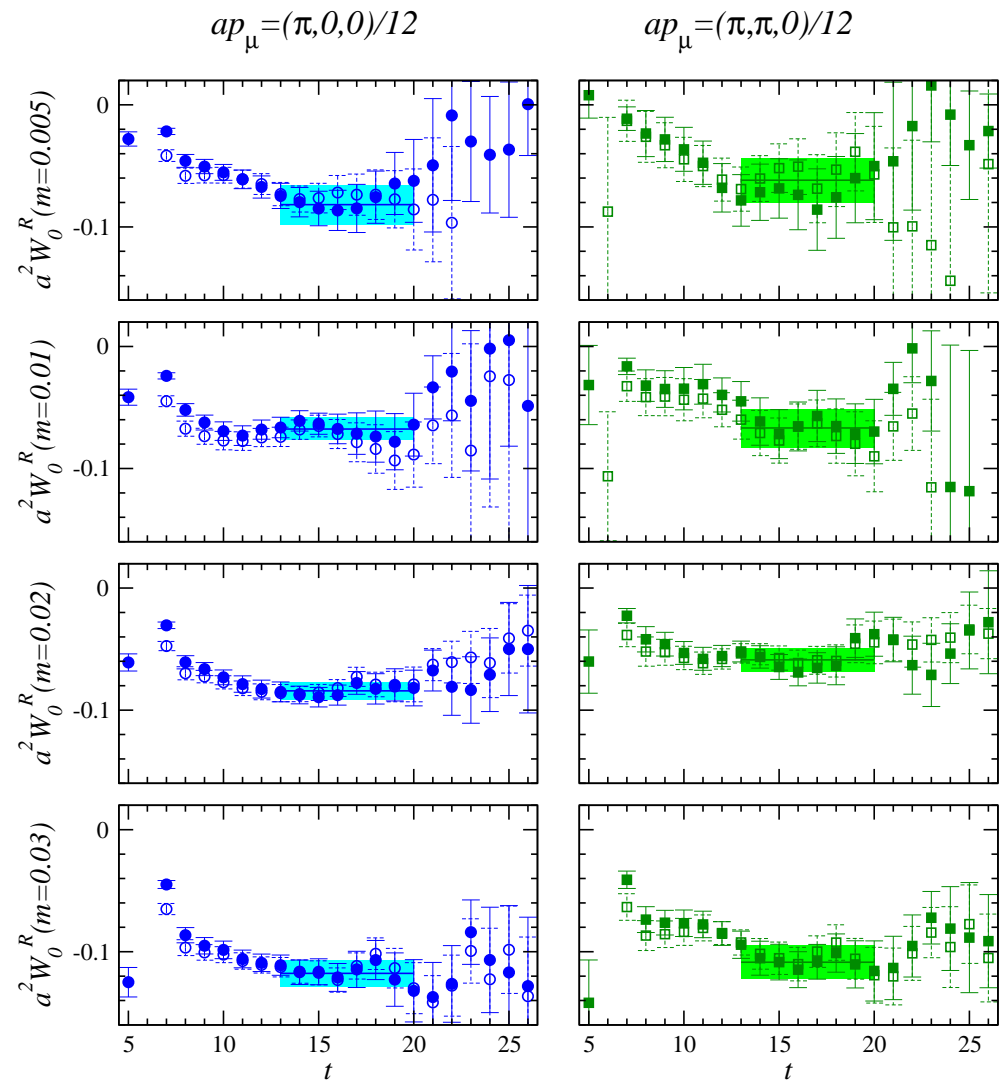

Figure 1: Time dependence of $W_{0}^{R}$ for $p \rightarrow \pi^{0}$ decay channel. Source of nucleon puts at $t=5$, and sink of $\pi^{0}$ is at $t=25$. Different symbols show the two different operator of interpolating nucleon field, which correspond to $\left(q^{T} C \gamma_{5} q\right) q$ (open) and $\left(q^{T} C \gamma_{4} \gamma_{5} q\right) q$ (filled) as defined in the context. The thick solid line indicates central value of constant fit combined two different operators between $13 \leq t \leq 20$ and its bound indicates 1-sigma error bound.

the fitting procedure and see the quality of fitting in the graph. In the chiral extrapolation of data at $q^{2}=0$ the fitting function is

$$
\begin{aligned}
f_{W_{0}}\left(\pi^{0}, \eta\right) & =a_{0}+a_{1}\left(m_{u d}+m_{\mathrm{res}}\right), \\
f_{W_{0}}\left(K^{0}, K^{+}\right) & =b_{0}+b_{1}\left(m_{u d}+m_{\mathrm{res}}\right)+b_{2}\left(m_{s}+m_{\mathrm{res}}\right) .
\end{aligned}
$$

To check the systematic uncertainty in chiral extrapolation, we compare two different maximum fitting ranges as $0.005 \leq m_{u d} \leq 0.03$ (4 masses) and $0.005 \leq m_{u d} \leq 0.02$ (3 masses). The linear function well describes the lattice results for each matrix elements even if we use four mass points. Actually the $\chi^{2} /$ dof for all matrix elements is below $\chi^{2} /$ dof $\simeq 2$.

\subsection{Final results}

Total systematic error is given by quadraticaly combining systematic errors of chiral extrapolation, lattice artifact and finite volume effect as shown in Table 1. Figure 2 plots comparison of finial results of twelve matrix elements with quenched (left panel) and indirect results (right panel) 

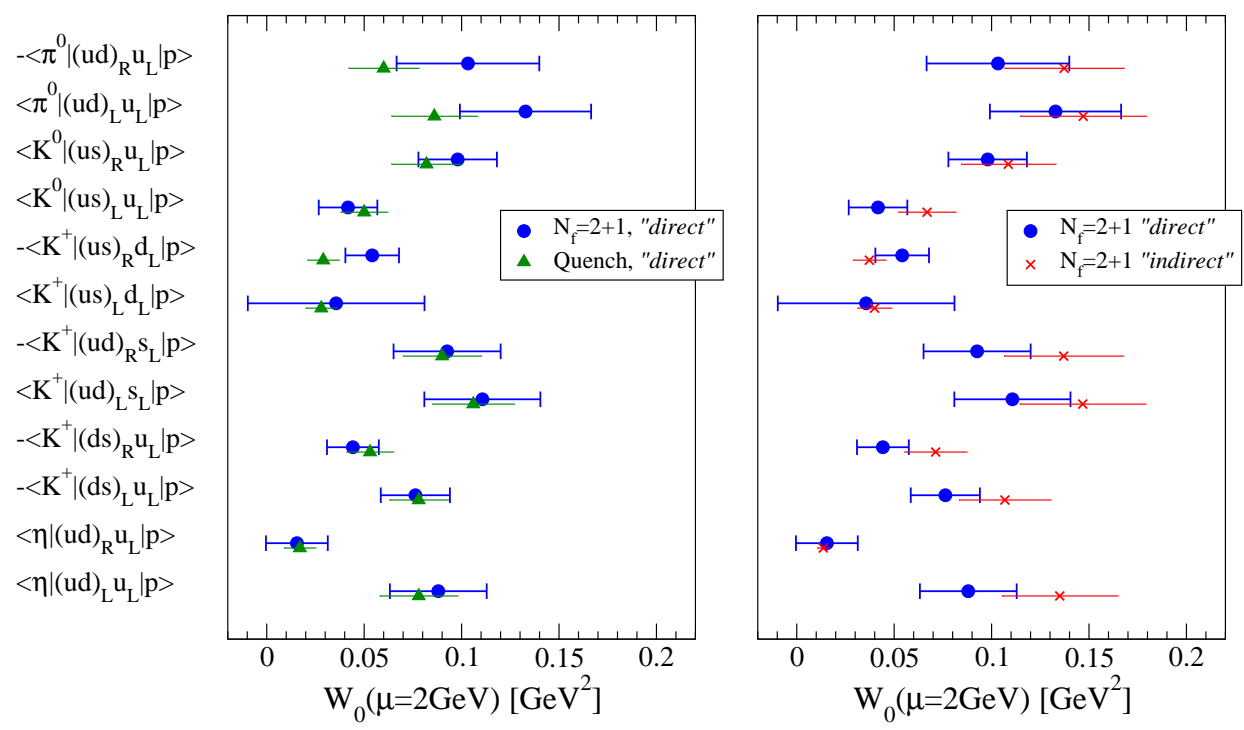

Figure 2: Summary of $W_{0}^{L / R}(\mu=2 \mathrm{GeV})$ for twelve independent matrix elements. Filled circles show the present results, and for the comparison the results in quenched QCD (open circle) and indirect method using chiral perturbation theory (cross) are plotted in the same raw.

which have been evaluated in $[6,8]$ respectively. This result is slightly changed from the quenched result, however these are consistent within $2 \sigma$ error. From the discrepancy between the quenched study and this work it turns out that $W_{0}$ slightly suffers a dynamical quark contribution although this contribution is within $1 \sigma$ error. We also note that there is no significant discrepancy between indirect method and direct one, contrary to conclusion in quenched study, however due to large uncertainty it is not able to clarify these comparison.

\section{Summary and discussion}

We present the lattice calculation of proton decay matrix elements using $2+1$ flavor dynamical domain-wall fermion configurations. The direct method using three-point function (nucleon)(operator)-(meson) in larger volume $L_{\sigma}^{3} \simeq 3 \mathrm{fm}^{3}$, is able to control several kinds of explicit and implicit uncertainties, especially remove the possibility of the significant effect of breaking of softpion theorem in the indirect calculation [8]. One is that, since our direct calculation does not need to assume soft-pion theorem, we directly evaluate hadronic contribution to matrix element involving individual operators and final meson state in lattice QCD. Comparing different fitting procedures as global or individual one when taking $q^{2}=0$ limit and physical point using lattice data, we carefully take account of systematic uncertainty of chiral extrapolation. Adding other systematic uncertainties, we obtain final results for twelve independent matrix elements in lattice QCD as shown in Figure 2. Our results have shown that there is no significant discrepancy between indirect-direct methods and also quenched-dynamical effects for all matrix elements, although the total error for these matrix elements is still large. Since the large uncertainties are due to fluctuation of three-point function in the lightest quark mass at large momentum, the reduction of such fluctuation is necessary using a further improvement of lattice data using larger volume or improved 
Table 1: Final results of renormalized $W_{0}^{L / R}(\mu=2 \mathrm{GeV})$ for individual matrix elements and error budget of statistical and systematic uncertainties. The first and second error in $W_{0}^{L / R}$ represent statistical and systematic error respectively. The third column shows the systematic error from chiral fitting, and fourth and fifth columns are uncertainties from lattice artifacts and finite volume effect. The final column shows the uncertainties included into lattice spacing $\left(\Delta a^{-1}\right)$ and renormalization factor $(\Delta Z)$.

\begin{tabular}{cccccc}
\hline \hline & $W_{0}(\mu=2 \mathrm{GeV}) \mathrm{GeV}^{2}$ & chiral fit & $\mathscr{O}\left(a^{2}\right)$ & $V$ & $\Delta a^{-1}+\Delta Z$ \\
\hline$-\left\langle\pi^{0}\left|(u d)_{R} u_{L}\right| p\right\rangle$ & $0.103(23)(27)$ & 0.025 & 0.010 & 0.006 & 0.004 \\
$\left\langle\pi^{0}\left|(u d)_{L} u_{L}\right| p\right\rangle$ & $0.133(29)(16)$ & 0.003 & 0.013 & 0.008 & 0.005 \\
$\left\langle K^{0}\left|(u s)_{R} u_{L}\right| p\right\rangle$ & $0.098(15)(12)$ & 0.003 & 0.010 & 0.006 & 0.004 \\
$\left\langle K^{0}\left|(u s)_{L} u_{L}\right| p\right\rangle$ & $0.042(13)(6)$ & 0.003 & 0.004 & 0.003 & 0.002 \\
$-\left\langle K^{+}\left|(u s)_{R} d_{L}\right| p\right\rangle$ & $0.054(11)(7)$ & 0.003 & 0.005 & 0.003 & 0.002 \\
$\left\langle K^{+}\left|(u s)_{L} d_{L}\right| p\right\rangle$ & $0.036(44)(9)$ & 0.008 & 0.004 & 0.002 & 0.001 \\
$-\left\langle K^{+}\left|(u d)_{R} s_{L}\right| p\right\rangle$ & $0.093(24)(11)$ & 0.003 & 0.009 & 0.006 & 0.003 \\
$\left\langle K^{+}\left|(u d)_{L} s_{L}\right| p\right\rangle$ & $0.111(22)(19)$ & 0.013 & 0.011 & 0.007 & 0.004 \\
$-\left\langle K^{+}\left|(d s)_{R} u_{L}\right| p\right\rangle$ & $0.044(12)(5)$ & 0.001 & 0.004 & 0.003 & 0.002 \\
$-\left\langle K^{+}\left|(d s)_{L} u_{L}\right| p\right\rangle$ & $0.076(14)(10)$ & 0.005 & 0.008 & 0.005 & 0.003 \\
$\left\langle\eta\left|(u d)_{R} u_{L}\right| p\right\rangle$ & $0.015(14)(6)$ & 0.006 & 0.002 & 0.001 & 0.001 \\
$\left\langle\eta\left|(u d)_{L} u_{L}\right| p\right\rangle$ & $0.088(21)(13)$ & 0.008 & 0.009 & 0.005 & 0.003 \\
\hline \hline
\end{tabular}

statistics in the future work. Our result may guarantee that proton lifetime can be evaluated with rigorous value including account of the non-perturbative contribution coming from hadronic effect, and further improved lattice result will be important to constrain several parameters in GUTs.

\section{Acknowledgments}

We thank other members of RBC-UKQCD collaboration for their valuable help with comments and encouragement, We also thank DOE USQCD and RIKEN BNL Research Center QCDOC computers at the Brookhaven National Laboratory. T. I. and A. S. is supported by U.S. DOE contract DE-AC02-98CH10886, and JSPS Grants ( 22540301, 23105714, 23105715 ) also support this work.

\section{References}

[1] H. Georgi, L. Glashow, Phys. Rev. Lett. 32, 438 (1974).

[2] J. Hisano, H. Murayama and T. Yanagida, Nucl. Phys. B 402, 46 (1993).

[3] H. Murayama, A. Pierce, Phys. Rev. D 65, 055009 (2002).

[4] H. Nishino, et al. (Super-Kamiokande Collaboration), Phys. Rev. Lett. 102, 141801 (2009).

[5] S. Aoki, et al. (JLQCD collaboration), Phys. Rev. D 62, 014506 (2000).

[6] Y. Aoki, C. Dawson, J. Noaki and A. Soni, (RBC collaboration) Phys. Rev. D 75, 014507 (2007).

[7] Y. Aoki, et al., (RBC-UKQCD collaboration), Phys. Rev. D 83, 074508 (2011).

[8] Y. Aoki, et al., (RBC-UKQCD collaboration), Phys. Rev. D 78, 054505 (2008). 